\title{
Outcome Analysis of Intracorneal Ring Segments for the Treatment of Keratoconus Based on Visual, Refractive, and Aberrometric Impairment
}

\author{
ALFREDO VEGA-ESTRADA, JORGE L. ALIO, LUIS F. BRENNER, JAIME JAVALOY, ANA BELEN PLAZA PUCHE, \\ RAFAEL I. BARRAQUER, MIGUEL A. TEUS, JOAQUIM MURTA, JORGE HENRIQUES, AND \\ ANTONIO UCEDA-MONTANES
}

- PURPOSE: To analyze the outcomes of intracorneal ring segment (ICRS) implantation for the treatment of keratoconus based on preoperative visual impairment.

- DESIGN: Multicenter, retrospective, nonrandomized study.

- METHODS: A total of 611 eyes of 361 keratoconic patients were evaluated. Subjects were classified according to their preoperative corrected distance visual acuity (CDVA) into 5 different groups: grade I, CDVA of 0.90 or better; grade II, CDVA equal to or better than 0.60 and worse than 0.90; grade III, CDVA equal to or better than 0.40 and worse than 0.60 ; grade IV, CDVA equal to or better than 0.20 and worse than 0.40; and grade plus, CDVA worse than 0.20. Success and failure indices were defined based on visual, refractive, corneal topographic, and aberrometric data and evaluated in each group 6 months after ICRS implantation.

- RESULTS: Significant improvement after the procedure was observed regarding uncorrected distance visual acuity in all grades $(P<.05)$. CDVA significantly decreased in grade $I(P<.01)$ but significantly increased in all other grades $(P<.05)$. A total of $37.9 \%$ of patients with preoperative CDVA 0.6 or better gained 1 or more lines of CDVA, whereas $82.8 \%$ of patients with preoperative CDVA 0.4 or worse gained 1 or more lines of CDVA $(P<.01)$. Spherical equivalent and keratometry readings showed a significant reduction in all grades $(P \leq .02)$. Corneal higher-order aberrations did not change after the procedure $(P \geq .05)$.

- CONCLUSIONS: Based on preoperative visual impairment, ICRS implantation provides significantly better results in patients with a severe form of the disease. A notable loss of CDVA lines can be expected in

Accepted for publication Aug 29, 2012.

From the Keratoconus Unit, Vissum Corporation, Alicante, Spain (A.V.E., J.L.A., L.F.B., J.J., A.B.P.P.); Division of Ophthalmology, Universidad Miguel Hernández, Alicante, Spain (A.V.E., J.L.A., J.J., A.B.P.P.); Centro de Oftalmología Barraquer (R.I.B.) and Institut Universitari Barraquer, Universitat Autònoma de Barcelona (R.I.B.), Barcelona, Spain; Vissum Corporation (M.A.T.) and University of Alcalá, Alcalá de Henares (M.A.T.), Madrid, Spain; Ophthalmology Department, Coimbra University Hospital, Coimbra, Portugal (J.M., J.H.); and Vissum Corporation, Sevilla, Spain (A.U.M.).

Inquiries to Jorge L. Alio, Avda de Denia s/n, Edificio Vissum, 03016 Alicante, Spain; e-mail: jlalio@vissum.com patients with a milder form of keratoconus. (Am J Ophthalmol 2013;155:575-584. (C) 2013 by Elsevier Inc. All rights reserved.)

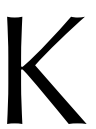
ERATOCONUS IS AN ECTATIC DEBILITATING corneal disorder characterized by a progressive corneal thinning that results in corneal protrusion, irregular astigmatism, and decreased vision. ${ }^{1}$ A variety of options have been described for the management of this pathologic condition, such as rigid gas-permeable contact lenses, ${ }^{2}$ corneal collagen cross-linking, ${ }^{3}$ intracorneal ring segment implantation, ${ }^{4-11}$ or keratoplasty. ${ }^{12}$

In a number of studies intracorneal ring segments (ICRS) have been demonstrated to be effective in improving visual acuity and reducing the refractive error and the mean keratometry in selected cases of keratoconic eyes. $^{4-11}$ Such a positive therapeutic effect is considered to be based on the induction of a remodeling of the corneal anterior and posterior surface's topography, which improves the optical quality of the cornea and reduces the optical aberrations, with consequent improvement in best-corrected visual acuity. ${ }^{4-11}$

Although in the healthy cornea, the sectorial arcuate addition to the corneal local volume caused by these implants at the corneal midperiphery induces a flattening of the central cornea in an arc-shortening effect, with the consequence of a decrease in myopic spherical equivalent attributable to the overall reduction in the optical power of the cornea, ${ }^{13}$ such corneal modeling effect may be different in structurally abnormal corneas such as in keratoconus. ${ }^{14}$

Even though the effectiveness of ICRS in the correction of keratoconus has been the subject of a number of studies and investigations, most reported evidence is based on the study of limited case series and may reflect only the experience of 1 surgeon or a limited and specific study group. Taking into consideration how diverse is the clinical condition of keratoconus in terms of anatomic severity and impact on the visual function, the reported results may be easily biased by the patient selection criteria, the degree of ectasia, the surgical technique employed, and the length of the follow-up, and thus may not reflect the general standards of the outcomes obtained with ICRS in the correction of keratoconus. 
We report herein the outcomes of ICRS in the surgical correction of different levels of severity of keratoconus obtained in a large multicenter series of cases analyzed based on a common definition of success regarding the visual outcomes.

\section{PATIENTS AND METHODS}

- PATIENTS: This multicenter, retrospective, interventional study comprised a total of 611 consecutive keratoconic eyes of 357 patients treated with ICRS implantation; 213 were male and 144 were female, ranging in age from 10 to 73 years (mean age: $35.15 \pm 11.62$ years). Before surgery each patient was exhaustively informed about the surgical procedure, its risks, and its benefits, and patients signed an informed consent in accordance with the Helsinki Declaration where they agreed that their clinical data may be included in scientific studies. The ethical board committee of Vissum Corporacion Oftalmologica Alicante approved the retrospective revision of the medical chart where were included the clinical data of the patients for developing this investigation. All patients were included after a retrospective review of all cases with the diagnosis of keratoconus in 6 different ophthalmologic centers that belong to the Spanish Network of Research in Ophthalmology (Red Tematica de Investigacion Cooperativa en Salud: Patologia Ocular del enevejecimiento, Calidad visual y Calidad de Vida, RD07/0062): 4 centers from the Vissum Corporation (Alicante, Madrid, Albacete, and Sevilla, all in Spain); the Barraquer Ophthalmological Center, Barcelona (Spain); and the Coimbra University Hospital (Portugal). All patients were included following the same protocol for data recording and analysis. Data recording was retrospective and was based on the review of all cases operated with ICRS implantation for correction of keratoconus in all participant centers from May 2000 to October 2011. Table 1 shows the contribution of each participating center in the current study. In those centers where more than 1 surgeon was involved in the surgical decision of the procedure, the number of surgeons involved is also mentioned.

Only keratoconus cases implanted with ICRS (KeraRing; Mediphacos, Belo Horizonte, Brazil; and Intacs; Addition Technology Inc, Fremont, California, USA) using either femtosecond laser technology or mechanical corneal dissection were included in this investigation. Patients with previous ocular surgery or an active ocular disease other than keratoconus were excluded from the study. Keratoconus diagnosis was based on corneal topography and slit-lamp observation. In all cases, preoperative findings characteristic of keratoconus were confirmed: corneal topography revealing an asymmetric bowtie pattern with or without skewed axes or keratoconus sign on slit-lamp examination, such as localized stromal thinning, conical
TABLE 1. Intracorneal Ring Segments for the Treatment of Keratoconus: Case Contribution of Each Participating Ophthalmology Center

\begin{tabular}{lc}
\hline \multicolumn{1}{c}{ Investigator/Center } & Eyes $(\mathrm{n})$ \\
\hline Vissum Corporation, Alicante, Spain & 296 (6 surgeons) \\
Coimbra University Hospital, Coimbra, & 112 (2 surgeons) \\
$\quad$ Portugal & \\
Vissum Corporation, Madrid, Spain & 93 (2 surgeons) \\
Centro de Oftalmologia Barraquer, & 59 (3 surgeons) \\
$\quad$ Barcelona, Spain & 30 (1 surgeon) \\
Vissum Corporation, Albacete, Spain & 21 (1 surgeon) \\
Vissum Corporation, Sevilla, Spain &
\end{tabular}

protrusion of the cornea at the apex, Fleischer ring, Vogt striae, or anterior stromal scar. ${ }^{1}$ In all cases ICRS implantation was indicated because of confirmed keratoconus diagnosis, poor motivation of the patient to wear contact lenses, or the existence of contact lens intolerance.

- GRADING SYSTEM ACCORDING TO THE LEVEL OF VISUAL LIMITATION: Patients were divided into 5 different groups according to the grading system based on the limitation of preoperative visual acuity. ${ }^{15}$

Grade I included patients with spectacle-corrected distance visual acuity (CDVA) (decimal notation) of 0.90 or better; grade II, patients with CDVA equal to or better than 0.60 and worse than 0.90 ; grade III, patients with CDVA equal to or better than 0.40 and worse than 0.60; grade IV, patients with CDVA equal to or better than 0.20 and worse than 0.40 ; and grade plus, patients with CDVA worse than 0.20 .

- EXAMINATION PROTOCOL: A comprehensive ophthalmologic examination was performed in all cases, which included uncorrected distance visual acuity (UDVA), CDVA, manifest refraction (sphere and cylinder), slit-lamp biomicroscopy, Goldmann tonometry, fundus evaluation, ultrasonic pachymetry, and corneal topographic analysis. As topographic data were collected from 6 different centers, a total of 3 different corneal topography systems were used for corneal examination: the CMS 100 Topometer (G. Rodenstock Instrument GmbH, Ottobrunn, Germany), CSO (CSO, Firenze, Italy), and Orbscan IIz system (Bausch \& Lomb, Rochester, New York, USA). The first 2 devices are Placido-based systems and the Orbscan IIz is a combined scanning-slit and Placidodisc topography system. Although the agreement between these specific devices has not been reported, Orbscan and Placido-based devices have been proven to provide similar accuracy and precision on calibrated spherical test surfaces. ${ }^{16}$ The following topographic data were evaluated and recorded with the 3 corneal topographic devices: corneal dioptric power in the flattest meridian for the $3-\mathrm{mm}$ central zone (K1), corneal dioptric power in the 
steepest meridian for the $3-\mathrm{mm}$ central zone (K2), and mean corneal power in the $3-\mathrm{mm}$ zone $(\mathrm{KM})$.

Corneal aberrometry was recorded and analyzed only for those patients examined with the CSO topography system (279 eyes), because this device was the only one with the capability to calculate directly this specific information. This topography system analyzes a total of 6144 corneal points of a corneal area enclosed in a circular annulus defined by an inner radius of $0.33 \mathrm{~mm}$ and an outer radius of $10 \mathrm{~mm}$ with respect to corneal vertex. The software of the CSO, the EyeTop2005 (CSO), automatically performs the conversion of corneal elevation profile into corneal wavefront data using the Zernike polynomials with an expansion up to the seventh order. In this study, the aberration coefficients and root mean square (RMS) values were calculated for a $6-\mathrm{mm}$ pupil in all cases. The corresponding RMS values were calculated for the following types of aberrations: higher-order (RMS HOA), coma-like (RMS coma-like) (computed for third-, fifth-, and seventh-order Zernike terms), and spherical-like (RMS sph-like) (computed for fourth- and sixth-order Zernike terms).

The patients wearing contact lenses were instructed in all cases to discontinue their use for at least 2 weeks before each examination for soft contact lenses and at least 4 weeks before each examination for rigid gas-permeable contact lenses.

All the clinical information from the ophthalmologic examinations was extracted from histories and recorded in a standardized database by 6 experienced optometrists following the same protocol.

- SURGICAL TECHNIQUE: Surgical procedures were performed by different surgeons, depending on each participating center in the study. In all cases an antibiotic prophylaxis consisting of topical ciprofloxacin was prescribed and taken every 8 hours for 2 days before surgery. All procedures were performed under topical anesthesia.

The mechanical surgical procedure was initiated by marking a reference point for centration (pupil center) and performing a radial incision of approximately $1.8 \mathrm{~mm}$ in length. After this, a calibrated diamond knife was set at approximately $70 \%$ of the mean corneal thickness, determined by ultrasonic pachymetry. From the base of the incision, pocketing hooks were used to create corneal pockets on each side of the incision, taking care to maintain a uniform depth. A device containing a semi-automated suction ring was placed around the limbus, guided by the previously marked reference point on the cornea. Two semicircular dissectors were then placed sequentially into the lamellar pocket to be steadily advanced by a rotational movement (counterclockwise and clockwise dissectors). In the femtosecond laser-assisted surgical procedure the suction ring was applied, and then the disposable glass lens of the laser system was applied first in order to applanate the cornea, fixate the eye, and help maintain a precise distance from the laser head to the focal point. ${ }^{7}$ Then, a continuous circular stromal tunnel was created at approximately $80 \%$ of corneal depth. The $60-\mathrm{kHz}$ IntraLase femtosecond system was always used (IntraLase Corp, Irvine, California, USA).

A total of 464 eyes $(75.80 \%)$ were operated with the femtosecond laser-assisted technique, whereas operations of the remaining 147 eyes (24.20\%) were performed with the mechanical dissection.

Regarding the ICRS type, Intacs (Addition Technology, Inc) were implanted in a total of 314 eyes $(51.45 \%)$, whereas KeraRings (Mediphacos) were implanted in 297 eyes $(48.55 \%)$.

The selection of the number ( 1 or 2$)$, arc length, and thickness of ICRS was performed following the nomogram defined by the manufacturer.

Postoperatively, topical tobramycin and dexamethasone eye drops (TobraDex; Alcon Laboratories Inc, Fort Worth, Texas, USA) were used every 6 hours for 1 week and then stopped. Topical lubricants were also prescribed every 6 hours for 1 month (Systane; Alcon Laboratories Inc).

- BEST-CASE GROUP: In order to carry out a more precise analysis of the results from the general series under study, a homogenous sample was taken where we included only the patients that underwent surgery with the femtosecond laser and were implanted with KeraRing ICRS, with selection criteria and surgery planning performed by the same experienced surgeon from the same specific center (J.L.A., Vissum, Alicante, Spain). This group comprised 114 eyes and was defined as the "best-case group." A statistical comparison of the variables under investigation between the general series and the "best-case group" was carried out in order to evaluate the representativeness of the population under study and the reliability of the results.

- DEFINITION OF SUCCESS AND FAILURE INDICES: The following criteria were defined for success and failure in order to evaluate the efficacy of the surgical procedure. Success was defined as those cases that showed 1 of the following characteristics 6 months after the procedure: (1) an improvement in 1 or more lines of uncorrected or corrected distance visual acuity, (2) a decrease in 2 or more diopters of spherical equivalent, (3) a decrease of at least $1 \mu \mathrm{m}$ of the RMS corneal higher-order or coma-like aberrations.

Failure was considered when 1 of the following criteria was found 6 months after the procedure: (1) a loss of 1 or more lines of uncorrected or corrected distance visual acuity, (2) an increase in 2 or more diopters of spherical equivalent, (3) an increase in $1 \mu \mathrm{m}$ or more in the RMS corneal higher-order or coma-like aberrations.

Patients who did not fulfill the change criteria outlined above were considered as remaining without significant change after the surgical procedure. 
- FOllOW-UP EVAlUATION: Data from the preoperative visit, the first postoperative day, and months 1, 3, and 6 were taken for the analysis of the results. On the first postoperative day, UDVA measurement and slit-lamp examination (intracorneal ring position and corneal integrity) were performed. Snellen chart UDVA and CDVA measurement, manifest refraction, slit-lamp examination, and corneal topography were performed in the rest of the postoperative examinations. A total of 268 eyes completed the 6-month follow-up evaluation.

- COMPLICATIONS: Complications after ICRS implantation for the treatment of keratoconus were not within the scope of the variables analyzed in the current investigation. Nevertheless, segments explanted for different reasons (segment migration, recurrent corneal erosion, corneal melting, corneal perforation, infectious keratitis, or patients unsatisfied because of poor refractive outcome) were observed in 38 of the 611 cases $(6.21 \%)$ under investigation. All these cases were excluded from the statistical analysis to avoid biased results and are the subject of an independent study previously published by our research group. ${ }^{17}$

- STATISTICAL ANALYSIS: The statistical analysis was performed using the SPSS software for Windows (version 15.0.1; SPSS Inc, Chicago, Illinois, USA). The mean values and standard deviations were calculated for every parameter during the follow-up. Normal distribution of all data samples was first checked by means of Kolmogorov-Smirnov test. If a parametric analysis was possible, the Student $t$ test for paired data was performed for comparisons between data obtained in the preoperative and postoperative examinations or consecutive postoperative visits. When a parametric analysis was not possible, the Wilcoxon rank sum test was applied to assess the significance of differences between preoperative and postoperative data, using in all instances the same level of statistical significance $(P<.05)$.

Regarding the comparisons among groups, the 1-way analysis of variance with the Bonferroni post hoc comparison procedure was used when parametric analysis was possible. If variances were not homogeneous (checked by the Levene test), the Tamhane post hoc analysis was used. When parametric analysis was not possible, the Kruskal-Wallis test was used, again using the same level of statistical significance $(P<.05)$. For post hoc analysis, the Mann-Whitney test with Bonferroni adjustment was used in order to avoid the experimental error rate in these cases.

- MAIN OUTCOMES MEASURES: Main outcome measures were visual acuity (UDVA, CDVA), manifest refraction, corneal topography, anterior corneal higher-order aberrations, and success and failure indices as described above.
TABLE 2. Percentage of Cases With a Loss of 2 or More Corrected Distance Visual Acuity Lines After Intracorneal Ring Segment Implantation

\begin{tabular}{lc}
\hline Keratoconus Grade & Lost $\geq 2$ lines CDVA \\
\hline I & $37.8 \%$ \\
II & $20.6 \%$ \\
III & $9.4 \%$ \\
IV & $4.6 \%$ \\
Plus & $3.7 \%$ \\
\hline
\end{tabular}

CDVA $=$ corrected distance visual acuity

\section{RESULTS}

THIS STUDY COMPRISED A TOTAL OF 611 CONSECUTIVE keratoconic eyes of 357 patients treated with ICRS; 213 were male and 144 were female, ranging in age from 10 to 73 years (mean age: $35.15 \pm 11.62$ years). A total of 268 eyes had a full ophthalmologic examination 6 months after the primary implantation of ICRS. According to the degree of visual limitation, of these 268 eyes, 37 eyes (13.80\%) were classified as grade I, 87 eyes $(32.46 \%)$ as grade II, 74 eyes (27.61\%) as grade III, 43 eyes $(16.04 \%)$ as grade IV, and 27 eyes $(10.07 \%)$ as grade plus.

There was no statistically significant difference when the results from the "best-case group" were compared with the results from the general series. Preoperatively, again, there was no statistically significant difference in terms of age $(P=.06)$, UDVA $(P=.83)$, spherical equivalent $(P=.57)$, CDVA $(P=.89)$, keratometry $(P>.05)$, and corneal aberrometry between groups $(P>.05)$. In addition, when we compared the postoperative outcomes of the variables mentioned above, among the subgroups defined according to the degree of visual limitation, we did not find any significant difference $(P>.05)$.

- VISUAL ACUITY: Patients classified as keratoconus grade I showed an increase in UDVA, from a preoperative mean value of $0.36 \pm 0.26$ to $0.45 \pm 0.24$ postoperatively $(P=.04)$. CDVA decreased significantly from a mean preoperative value of $0.97 \pm 0.06$ to a mean postoperative value of $0.86 \pm 0.18(P<.01)$. In this group, $37.8 \%(14 / 37)$ of patients lost 2 or more lines of CDVA (Table 2). In patients with keratoconus grade II, UDVA significantly improved from a mean preoperative value of $0.27 \pm 0.21$ to a postoperative value of $0.44 \pm 0.24(P<.01)$. CDVA also increased from a preoperative mean value of $0.71 \pm 0.08$ to $0.75 \pm 0.22$ postoperatively $(P=.04)$. In this group, $20.6 \%(18 / 87)$ of patients lost 2 or more lines of CDVA (Table 2). In patients with keratoconus grade III, UDVA and CDVA increased significantly from a preoperative mean level of $0.16 \pm 0.14$ and $0.45 \pm 0.53$, respectively, to a postoperative mean level of $0.24 \pm 0.16$ and $0.57 \pm 0.22$, respectively $(P<.01)$. In this group, 
TABLE 3. Visual Outcomes 6 Months After Intracorneal Ring Segment Implantation: Comparison of Gained and Lost Corrected Distance Visual Acuity Lines Between the Least and the Most Advanced Cases of Keratoconus

\begin{tabular}{lccc}
\hline \multicolumn{1}{c}{ Visual Acuity } & $\begin{array}{c}\text { Gained } \geq 1 \\
\text { Line CDVA }\end{array}$ & $\begin{array}{c}\text { Lost } \geq 1 \\
\text { Line CDVA }\end{array}$ & $\begin{array}{c}\text { Lost } \geq 2 \\
\text { Lines CDVA }\end{array}$ \\
\hline CDVA $\geq 0.6$ grade I + II & $37.90 \%$ & $36.29 \%$ & $25.80 \%$ \\
CDVA $\leq 0.4$ grade IV + plus & $82.85 \%$ & $10.00 \%$ & $4.28 \%$ \\
$P$ value & $<.01$ & $<.01$ & $<.01$ \\
\hline
\end{tabular}

CDVA = corrected distance visual acuity.

9.45\% (7/74) of patients lost 2 or more lines of CDVA (Table 2). Patients with keratoconus grade IV also showed a statistically significant improvement of both UDVA and CDVA from preoperative values of $0.13 \pm 0.09$ and $0.27 \pm$ 0.05 , respectively, to a postoperative level of $0.20 \pm 1.55$ and $0.50 \pm 0.22$, respectively $(P<.01)$. In this group $4.65 \%(2 / 43)$ of patients lost 2 or more lines of CDVA (Table 2). Finally, patients classified as keratoconus grade plus are the ones that showed the largest increase in CDVA. Preoperatively these patients had a mean CDVA of $0.09 \pm 0.05$, which improved to a postoperative value of $0.38 \pm 0.26(P<.01)$. UDVA also increased significantly from a preoperative $0.05 \pm 0.04$ to postoperative $0.14 \pm 0.14(P=.03)$. In grade plus, only $3.7 \%(1 / 27)$ of patients lost 2 or more lines of CDVA (Table 2).

Comparison of the eyes with the least advanced keratoconus (grades I and II) and eyes with the most advanced keratoconus (grades IV and plus) showed statistically significant difference in relation to the cases that lost 2 or more lines of CDVA $(P<.01)$. Thus, $25.8 \%(32 / 124)$ of patients with CDVA 0.6 or better (grades I and II) presented a loss of 2 or more lines of CDVA, whereas just $4.2 \%(3 / 70)$ of patients with CDVA 0.4 or worse (grades IV and plus) showed a loss of 2 or more lines of CDVA (Table 3).

- SPHERICAL EQUIVALENT AND KERATOMETRY: Analysis of the mean spherical equivalent (SE) showed a statistically significant reduction in all grades of keratoconus 6 months after the primary surgery $(P \leq .02)$. Patients in grade I showed a decrease from a preoperative mean SE of $-2.86 \pm 2.68$ to a mean postoperative SE of $-1.76 \pm$ $2.57(P<.01)$. In grade II, preoperative SE was $-3.88 \pm$ 3.58 , decreasing to a postoperative level of $-2.07 \pm 2.68$ $(P<.01)$. Patients in grade III presented a reduction from a preoperative mean value of $-5.25 \pm 4.33$ to a postoperative mean SE of $-2.82 \pm 4.06(P<.01)$. In grade IV, SE decreased from a preoperative mean value of $-6.35 \pm$ 5.04 to a postoperative value of $-4.18 \pm 5.42(P<.01)$, and in grade plus, patients showed a reduction from preoperative $-7.43 \pm 6.10$ to postoperative $-3.93 \pm 5.63(P=.02)$. Patients with the most advanced form of the disease (grades
IV and plus) showed the largest reduction in terms of spherical equivalent in comparison with patients with the least advanced form of keratoconus (grades I and II) $(P=.04)$.

A statistically significant reduction of the flattest, steepest, and mean keratometry readings was also observed in all grades $(P \leq .01)$ (Table 4$)$. Patients classified as grade I showed a reduction in the mean keratometry of 1.55 diopters (D). In grade II, reduction of the mean keratometry was 1.72 D. Patients in grade III exhibited a decrease of $2.84 \mathrm{D}$ in the mean keratometry. Patients grouped under grade IV showed a reduction in the mean keratometry of $4.01 \mathrm{D}$. Finally, the largest reduction was observed in the more advanced cases (grade plus), where patients showed a decrease of $5.61 \mathrm{D}$ in the mean keratometry.

- ANTERIOR CORNEAL HIGHER-ORDER ABERRATIONS: Analysis of anterior corneal HOA showed that even when there was a postoperative reduction in all types of aberrations under investigation, only the RMS coma-like in grade III was within the limit of statistical significance $(P=.05)$. All other values did not show a significant change $(P>.05)$ (Table 5). Comparison of the results according to the visual limitation between the groups with the least advanced form of the disease (grades I and II) and the group with the most advanced form of the disease (grades IV and plus) showed statistically significant better results in favor of the patients with a more advanced form of the disease $(P<.01)$, in terms of the aberrometric data analyzed.

- SUCCESS AND FAILURE INDICES ACCORDING TO VISUAL LIMITATION: As mentioned previously, success and failure indices were defined in order to better evaluate the efficacy of the surgical procedure, according to the degree of the preoperative visual disability of the patients.

Tables 6 and 7 show the distribution of the cases according to the success and failure indices. Table 8 reports the percentage of cases that gained or lost 1 or more lines of CDVA according to the preoperative visual limitation. This table demonstrates that the groups with more severe keratoconus are the ones that gain more lines of CDVA and, on the other hand, are the ones with the lesser number of patients losing lines of CDVA.

Comparison of the eyes with mild keratoconus (grades I and II) and the eyes with severe keratoconus (grades IV and plus) shows a statistically significant difference regarding the number of cases that lost or gained 1 or more lines of CDVA $(P<.01)$. Thus, 37.9\% (47/124) of patients with CDVA 0.6 or better (grades I and II) gained 1 or more lines of CDVA, whereas $82.8 \%$ (58/70) of patients with CDVA 0.4 or worse (grades IV and plus) gained 1 or more lines of CDVA (Table 3). In addition, 36.3\% (45/124) of patients who had CDVA of 0.6 or better lost 1 or more lines of CDVA, whereas just 10\% (7/70) of patients with CDVA of 0.4 or worse lost 1 or more lines of CDVA (Table 3 ). 


\begin{tabular}{ccccccccccc}
\hline \multicolumn{7}{c}{ TABLE 4. Changes in Keratometry Readings After Intracorneal Ring Segment Implantation in Cases of Keratoconus Classified } \\
According to the Preoperative Visual Limitation
\end{tabular}

\begin{tabular}{|c|c|c|c|c|c|c|c|c|c|}
\hline Keratoconus Grade & RMS-HO Pre $(\mu \mathrm{m})$ & RMS-HO $6 \mathrm{M}(\mu \mathrm{m})$ & $P$ Value & RMS-ComL Pre & RMS-ComL 6M & $P$ Value & RMS-SphL Pre & RMS SphL 6M & $P$ Value \\
\hline I & $\begin{array}{r}2.26 \pm 1.28 \\
(0.33-5.10)\end{array}$ & $\begin{array}{r}1.80 \pm 0.93 \\
(0.75-4.60)\end{array}$ & .69 & $\begin{array}{r}2.07 \pm 1.20 \\
(0.17-4.86)\end{array}$ & $\begin{array}{r}1.60 \pm 0.94 \\
(0.58-4.54)\end{array}$ & .97 & $\begin{array}{l}0.85 \pm 0.51 \\
\quad(0.11-2.43)\end{array}$ & $\begin{array}{r}0.74 \pm 0.36 \\
(0.26-1.59)\end{array}$ & .64 \\
\hline II & $\begin{array}{l}3.26 \pm 1.79 \\
(0.71-14.19)\end{array}$ & $\begin{array}{r}3.01 \pm 1.53 \\
(0.94-7.21)\end{array}$ & .15 & $\begin{array}{l}2.97 \pm 1.52 \\
(0.52-10.89)\end{array}$ & $\begin{array}{r}2.76 \pm 1.46 \\
(0.80-6.84)\end{array}$ & .07 & $\begin{array}{r}1.19 \pm 1.14 \\
(0.24-9.09)\end{array}$ & $\begin{array}{c}1.10 \pm 0.64 \\
(0.23-3.46)\end{array}$ & .23 \\
\hline III & $\begin{array}{c}4.20 \pm 2.39 \\
\quad(1.31-13.19)\end{array}$ & $\begin{array}{r}3.84 \pm 1.72 \\
\quad(1.42-8.19)\end{array}$ & .15 & $\begin{array}{l}3.86 \pm 2.23 \\
\quad(1.22-12.86)\end{array}$ & $\begin{array}{l}3.47 \pm 1.60 \\
(1.35-7.78)\end{array}$ & .05 & $\begin{array}{r}1.46 \pm 1.17 \\
(0.27-6.87)\end{array}$ & $\begin{array}{r}1.53 \pm 0.89 \\
(0.44-3.85)\end{array}$ & .40 \\
\hline IV & $\begin{array}{r}4.03 \pm 1.95 \\
(1.26-8.32)\end{array}$ & $\begin{array}{l}3.43 \pm 2.08 \\
\quad(1.62-10.39)\end{array}$ & .70 & $\begin{array}{r}3.70 \pm 1.91 \\
(0.40-7.95)\end{array}$ & $\begin{array}{c}3.00 \pm 2.02 \\
(1.16-9.35)\end{array}$ & .43 & $\begin{array}{r}1.44 \pm 0.83 \\
(0.33-4.83)\end{array}$ & $\begin{array}{r}1.52 \pm 0.85 \\
(0.46-4.54)\end{array}$ & .18 \\
\hline Plus & $\begin{array}{c}6.03 \pm 4.02 \\
\quad(1.84-20.71)\end{array}$ & $\begin{array}{r}4.60 \pm 2.72 \\
(2.26-9.39)\end{array}$ & .11 & $\begin{array}{l}5.53 \pm 3.45 \\
\quad(1.79-16.20)\end{array}$ & $\begin{array}{r}4.10 \pm 2.56 \\
(1.87-9.19)\end{array}$ & .11 & $\begin{array}{l}2.16 \pm 2.33 \\
\quad(0.42-12.91)\end{array}$ & $\begin{array}{r}1.84 \pm 1.37 \\
(0.71-5.28)\end{array}$ & .20 \\
\hline
\end{tabular}

ComL = coma-like aberration; $\mathrm{HO}=$ higher-order aberration; Pre = preoperative period; RMS = root mean square; SphL $=\mathrm{spherical-like}$ aberration; $6 \mathrm{M}=6$ months postoperative period.

\section{DISCUSSION}

IN THIS STUDY WE EVALUATED THE OUTCOMES OF ICRS implantation for the management of keratoconus based on patients' preoperative visual impairment.

Several grading systems have been described in the literature in order to classify the severity of keratoconus. ${ }^{18}$ Nevertheless, most of these grading systems have been developed taking into account the topographic morphology of the disease, the corneal keratometry readings, and corneal aberrometry, without considering other clinical data that are closely related to the visual disability caused by keratoconus. Thus, the success or failure of this surgical technique is analyzed in this study, using a keratoconus classification based on the functional performance of the patient's visual system, ${ }^{15}$ such as visual acuity, rather than just the geometric assessment of the cornea, which is unpredictable because of response of keratoconic eyes. ${ }^{19-21}$

Most of the published studies analyzing the effectiveness of ICRS implantation for the treatment of keratoconus agree that there is a significant flattening of the central cornea after the procedure. ${ }^{4-8,22-25}$ Our results confirm that finding, as we found a statistically significant reduction in the keratometry readings in all grades of keratoconus. We observed that the largest reduction in mean keratometry was found in those cases with the most severe disease. In addition, patients classified under this category were those who achieved the greatest decrease in spherical equivalent, which correlates well with the amount of flattening in the central cornea. Ertan and Kamburoglu ${ }^{6}$ conducted a study with a large keratoconic population where they also found that patients with severe keratoconus were the ones in whom the largest reduction of 
TABLE 6. Percentage Change in Success Indices 6 Months After Implanting Intracorneal Ring Segments in Cases of Keratoconus Classified According to Preoperative Visual Impairment

\begin{tabular}{lccccc}
\hline Keratoconus Grade & Gain $\geq 1$ Line UDVA & Decrease $\geq 2$ D SE & Gain $\geq 1$ Line CDVA & Decrease $\geq 1 \mu \mathrm{m}$ RMS-HO $(\mu \mathrm{m})$ & Decrease $\geq 1 \mu \mathrm{m} \mathrm{RMS}$-ComL \\
\hline I & $65.51 \%$ & $36.11 \%$ & $13.51 \%$ & $21.05 \%$ & $21.05 \%$ \\
II & $67.21 \%$ & $40.96 \%$ & $49.42 \%$ & $16.21 \%$ & $18.91 \%$ \\
III & $63.46 \%$ & $50.00 \%$ & $54.05 \%$ & $25.00 \%$ & $28.57 \%$ \\
IV & $73.07 \%$ & $59.52 \%$ & $81.39 \%$ & $29.41 \%$ & $23.52 \%$ \\
Plus & $77.77 \%$ & $60.00 \%$ & $85.18 \%$ & $20.00 \%$ & $20.00 \%$ \\
\hline
\end{tabular}

$\mathrm{CDVA}=$ corrected distance visual acuity; ComL = coma-like aberration; $\mathrm{D}=$ diopters; $\mathrm{HO}=$ higher-order aberration; $\mathrm{RMS}=$ root mean square; $\mathrm{SE}=$ spherical equivalent; UDVA = uncorrected distance visual acuity.

TABLE 7. Percentage Changes in Failure Indices 6 Months After Implanting Intracorneal Ring Segments in Cases of Keratoconus Classified According to Preoperative Visual Impairment

\begin{tabular}{lccccc}
\hline Keratoconus Grade & Lost $\geq 1$ Line UDVA & Increase $\geq 2$ D SE & Lost $\geq 1$ line CDVA & Increase $\geq 1 \mu \mathrm{m}$ RMS-HO $(\mu \mathrm{m})$ & Increase $\geq 1 \mu \mathrm{m}$ RMS ComL \\
\hline I & $14 \%$ & $5.55 \%$ & $51.00 \%$ & $10.52 \%$ & $10.52 \%$ \\
II & $29.5 \%$ & $10.84 \%$ & $29.88 \%$ & $8.1 \%$ & $8.1 \%$ \\
III & $21.15 \%$ & $12.00 \%$ & $18.91 \%$ & $14.28 \%$ & $17.85 \%$ \\
IV & $15.38 \%$ & $7.14 \%$ & $9.30 \%$ & $5.88 \%$ & $5.88 \%$ \\
Plus & $5.55 \%$ & $12.00 \%$ & $11.1 \%$ & $0 \%$ & $0 \%$
\end{tabular}

CDVA = corrected distance visual acuity; ComL = coma-like aberration; $\mathrm{D}=$ diopter; $\mathrm{HO}=$ higher-order aberration; $\mathrm{RMS}=$ root mean square; $\mathrm{SE}=$ spherical equivalent; UDVA = uncorrected distance visual acuity.

\begin{tabular}{|c|c|c|}
\hline \multicolumn{3}{|c|}{$\begin{array}{c}\text { TABLE 8. Percentage of Cases That Gained or Lost } 1 \text { or } \\
\text { More Lines of Corrected Distance Visual Acuity } 6 \text { Months } \\
\text { After Intracorneal Ring Segment Implantation in Cases of } \\
\text { Keratoconus Classified According to the Preoperative } \\
\text { Visual Impairment }\end{array}$} \\
\hline Keratoconus Grade & Gained $\geq 1$ Line CDVA & Lost $\geq 1$ Line CDVA \\
\hline 1 & $13.51 \%$ & $51.00 \%$ \\
\hline$\|$ & $49.42 \%$ & $29.88 \%$ \\
\hline III & $54.05 \%$ & $18.91 \%$ \\
\hline IV & $81.39 \%$ & $9.30 \%$ \\
\hline Plus & $85.18 \%$ & $11.1 \%$ \\
\hline
\end{tabular}

the keratometry was observed. Furthermore, in another study, Boxer Wachler and associates ${ }^{21}$ found, again, that the greatest reduction in the spherical equivalent was found in those patients with the more advanced form of the disease.

In the current study, we found a statistically significant improvement in the UDVA in all grades of keratoconus analyzed. We observed that the most significant improvement was achieved by the group of patients classified under the category of grade plus. Most of the studies that have evaluated the impact of ICRS in the management of keratoconus patients reported a significant improvement in the
UDVA..$^{4-6,8,10,21,25-27}$ In addition, studies that have analyzed the clinical outcomes according to the severity of the disease also reported a significant improvement in patients who suffered from a severe form of keratoconus. ${ }^{5,21,27}$ By analyzing the results according to the degree of visual limitation, we found that more than $65 \%$ of our patients gain at least 1 line of UDVA after the primary implantation of the ICRS. These results are consistent with those found in the literature, as most of the studies that have been carried out reported a gain of lines in UDVA. ${ }^{6,7,10,21,27,28}$ We observed that the most significant increase in the UDVA was found in patients classified as grade plus, where more than $77 \%$ gained 1 or more lines of UDVA. Furthermore, in this group of patients we observed the least number of eyes losing 1 or more lines of UDVA (Tables 6 and 7).

The patients evaluated in the present study were classified according to preoperative CDVA. There are several publications in the literature showing that ICRS implantation in keratoconus eyes results in an improvement of the CDVA. ${ }^{4,5,8,11,20-24,29}$ However, in the present study, patients with the milder form of the disease (patients classified as grade I) showed a statistically significant decrease in the CDVA. This might be related to the fact that in those investigations, the analysis of the outcomes regarding CDVA was performed evaluating all the population under study as a whole, without considering 
the degree of visual impairment. Thus we can observe that, when we analyzed the outcomes of patients implanted with ICRS for the management of keratoconus based on a grading system that takes into account the preoperative corrected visual acuity, the results are somewhat different from those previously reported by other authors. We found that there is a clear correlation between the degree of preoperative visual impairment of the patients and the change in the CDVA achieved after the procedure. Thus, when we analyzed what we defined as "success index," we found that patients presenting the worst preoperative best-corrected visual acuity are the ones who gain more lines of CDVA after the ICRS implantation (Tables 6 and 8). The same behavior was found when we evaluated the failure index related to CDVA. Thus, patients who have the greatest levels of CDVA during the preoperative examination are the ones showing more loss of CDVA lines after the procedure (Tables 7 and 8 ). Furthermore, if we compare the outcomes of the patients with the least advanced keratoconus (grades I and II) with the eyes with more advanced disease (grades IV and plus), the latter group has more than $82 \%$ of cases showing a gain of 1 or more lines of CDVA after the surgery, whereas less than $40 \%$ will do so in the least advanced group. In addition, $10 \%$ of patients showed a loss of 1 or more lines of CDVA, and $4.2 \%$ of patients 2 or more lines, in the group with the more advanced form of the disease. On the other hand, this percentage is significantly higher in patients with the least advanced form of the disease, with $36.2 \%$ and $25.8 \%$ losing 1 or 2 or more lines of CDVA, respectively (Table 3 ). Thus, the analysis of outcomes considering the degree of the preoperative visual limitation clearly indicates that patients who benefit most from ICRS implantation for the management of keratoconus are the ones with the most advanced form of the disease. Furthermore, there is clear evidence of the poor outcomes that are achieved after implanting ICRS in patients in whom the corrected visual acuity before the procedure is not compromised, and for this reason such a surgical procedure should not be considered as a therapeutic approach in this group of patients. However, there is a factor that we did not analyze in the present report, which is tolerance to contact lenses. Contact lens tolerance might be improved after ICRS implantation in this group of patients, as suggested by other authors. ${ }^{5,30}$ Nevertheless, further studies with a larger sample of patients and analysis of the results according to the preoperative degree of visual impairment should be performed in order to address this issue.

As previously mentioned, we observed that patients classified as having grade I keratoconus showed a significant reduction in the CDVA in spite of the improvement of mean preoperative spherical equivalent, UDVA, and significant reduction in keratometric readings, with no significant changes in the corneal higher-order aberrations. This fact is probably related to biomechanical changes induced by the ICRS that may lead to a positive influence in the topographic morphology of the cornea, thus improving the refractive error, but with a negative impact in the visual function of the patient. Another hypothesis that could explain this behavior is that introducing a synthetic element inside the stroma and modifying the distribution of the corneal lamellae will lead to an unpredictable change in the refractive index of the cornea, thus affecting the CDVA in this group of patients. However, an appropriate analysis of the corneal biomechanics behavior and changes in the refractive index of the cornea should be performed in these cases in order to confirm this evidence. In addition, factors that may be related to these findings are that patients with a good corrected visual acuity are the ones who are more prone to lose lines of vision, as they have not "too much to gain" but "too much to lose." Indeed, it is because of these observations that we consider that ICRS implantation is not the best indication in patients in whom corrected visual acuity is not compromised.

In the present study, $21.2 \%$ of patients remained without significant change regarding a loss or gain in lines of CDVA. This group of patients is considered to be without benefit or harm after implanting ICRS. The heterogeneous variables analyzed in the present series, such as different surgeons, different types of ICRS, and different types of surgical procedures, may have an impact on the results observed in this group of patients. Another explanation could be the use of single or double segments depending on the preoperative topographic position of the ectasia. Although in the present study the number of segments to be implanted was decided based on the nomogram provided by the manufacturer, some studies have demonstrated that implanting a single segment may provide better results than implanting 2 segments in cases of peripheral ectasia and that implanting 2 segments can lead to clinical improvement in cases of keratoconus, with central steepening in the topographic image. ${ }^{29}$ In spite of the above-mentioned comments, when we analyzed patients grouped with the milder form of the disease (grades I and II) and compared the results with those classified under the category of the most severe form (grades IV and plus), we observed the same trend in relation to the outcomes in each group. We found that $25.8 \%$ of cases in grades I and II remained without a significant change of CDVA in comparison to only $7.1 \%$ of patients in grade IV and grade plus. This observation will also support the evidence that patients who benefit the most from implanting ICRS for the correction of keratoconus are those with the most advanced form of the disease.

In the current investigation we also evaluated the behavior of the anterior corneal higher-order aberrations according to the degree of visual impairment after ICRS implantation. We found that there was no statistically significant difference between the preoperative and the postoperative period in terms of corneal aberrometric 
data. There are some studies published in the literature that have analyzed the change in corneal aberrations induced by this surgery, and they have found similar results. ${ }^{25,31,32}$ However, even when we did not find any statistically significant change in any of the corneal aberrations under study in any of the grades of keratoconus, the group of patients where the largest decrease in terms of RMS HOA, RMS coma-like, and RMS sph-like was achieved happened in the most advanced cases (grade IV and grade plus). These findings have been previously reported by our research group, ${ }^{7}$ where we found that the higher the preoperative RMS value of the cornea, the higher the amount of reduction can be achieved after ICRS implantation, specifically in terms of RMS HOA and RMS coma-like aberrations.

The retrospective nature of this study; the different surgical techniques for implanting the ICRS employed, such as mechanical and femtosecond-assisted; the significant rate of dropouts during the follow-up period; and the different types of ICRS implanted are, among others, the major drawbacks of the current study. Nevertheless, we must remember that previous reports have shown that there are no statistically significant differences in the refractive outcomes when comparing the results of mechanical and femtosecond laser-assisted ICRS implantation technique. ${ }^{30}$ Other publications have also found no significant difference when comparing the efficacy of different types of ICRS. ${ }^{31,33}$ In addition, even when we had a significant amount of dropouts during the follow-up period, we believe that this should not be a factor biasing our results as we evaluated almost 300 cases with a follow-up period of 6 months. Furthermore, in order to evaluate the reliability of our results from such a heterogeneous population, we decided to take a representative sample that was defined as the "best-case group," where we included patients who were operated by the same experienced surgeon, using the same surgical technique (femtosecond-assisted) and the same type of ICRS. The results obtained in this group of patients were found to be statistically comparable with the ones obtained in the general population included in all the variables evaluated. In addition, and even when several ophthalmologists were involved in the surgical planning of ICRS implantation in the general series, the results obtained by a single surgeon (best case group) were statistically comparable with those obtained by the total of surgeons who participated in the study. This fact demonstrates that the results shown by 1 surgeon represent the outcome trends of a surgical practice corresponding to the whole sample of surgeons from this series.

The milestone of every ophthalmic procedure should focus on improving and not on deteriorating the visual function of the patient. For this reason, and after a thorough analysis of the results that we present in the current investigation, we conclude that implantation of ICRS for the management of keratoconus should be kept mainly for those cases that have a clear impairment of visual performance. In summary, based on the outcomes of the present investigation we would not advise this procedure in patients in whom corrected visual acuity is not compromised, at least until new studies demonstrate that tolerance to contact lenses can be restored in this group of patients, new nomograms of implantation are developed, and a better understanding of corneal biomechanics allows us to offer the patient a better therapeutic approach in order to improve his or her visual function. Those patients with more advanced keratoconus are those who are more prone to obtain a positive outcome following ICRS implantation. Further investigations are required for a better indication for ICRS in patients with early keratoconus or those in whom visual function is not significantly affected.

ALL AUTHORS HAVE COMPLETED AND SUBMITTED THE ICMIE FORM FOR DISCLOSURE OF POTENTIAL CONFLICTS OF INTEREST. The authors do not have any affiliations with or involvement in any organization or entity with a direct financial interest in the subject matter or materials discussed in the manuscript. This study has been supported in part by a grant from the Spanish Ministry of Health, Instituto Carlos III, Red Temática de Investigación Cooperativa en Salud "Patología ocular del envejecimiento, calidad visual y calidad de vida," Subproyecto de Calidad Visual (RD07/0062), Madrid, Spain. Contributions of authors: involved in design and conduct of the study (A.V.E., J.L.A.); data collection and management (A.V.E., L.F.B., J.J., A.B.P., R.I.B., M.A.T., J.M., J.H., A.U.); analysis and interpretation of the data (A.V.E., J.L.A., L.F.B., J.J.); writing and preparation of the manuscript (A.V.E.); and review and approval of the manuscript (A.V.E., J.L.A., L.F.B., J.J., A.B.P., R.I.B., M.A.T., J.M., J.H., A.U.).

\section{REFERENCES}

1. Rabinowitz YS. Keratoconus. Surv Ophthalmol 1998;42(4): 297-319.

2. Garcia-Lledo M, Feinbaum C, Alió JL. Contact lens fitting in keratoconus. Compr Ophthalmol Update 2006;7(2):47-52.

3. Wollensak G, Spoerl E, Seiler T. Riboflavin/ultraviolet-ainduced collagen crosslinking for the treatment of keratoconus. Am J Ophthalmol 2003;135(5):620-627.

4. Coskunseven E, Kymionis GD, Tsiklis NS, et al. One-year results of intrastromal corneal ring segment implantation
(KeraRing) using femtosecond laser in patients with keratoconus. Am J Ophthalmol 2008;145(5):775-779.

5. Shetty R, Kurian M, Anand D, Mhaske P, Narayana KM, Shetty BK. Intacs in advanced keratoconus. Cornea 2008; 27(9):1022-1029.

6. Ertan A, Kamburoglu G. Intacs implantation using femtosecond laser for management of keratoconus: comparison of 306 cases in different stages. J Cataract Refract Surg 2008;34(9):1521-1526.

7. Shabayek MH, Alió JL. Intrastromal corneal ring segment implantation by femtosecond laser for keratoconus correction. Ophthalmology 2007;114(9):1643-1652. 
8. Kymionis GD, Siganos CS, Tsiklis NS, et al. Long-term follow-up of Intacs in keratoconus. Am J Ophthalmol 2007; 143(2):236-244.

9. Alió JL, Shabayek MH, Artola A. Intracorneal ring segments for keratoconus correction: long-term follow-up. J Cataract Refract Surg 2006;32(6):978-985.

10. Colin J. European clinical evaluation: use of Intacs for the treatment of keratoconus. J Cataract Refract Surg 2006; 32(5):747-755.

11. Kanellopoulos AJ, Pe LH, Perry HD, Donnenfeld ED. Modified intracorneal ring segment implantations (INTACS) for the management of moderate to advanced keratoconus: efficacy and complications. Cornea 2006;25(1):29-33.

12. Sutton $\mathrm{G}$, Hodge $\mathrm{C}, \mathrm{Mc}$ Ghee $\mathrm{CN}$. Rapid visual recovery after penetrating keratoplasty for keratoconus. Clin Experiment Ophthalmol 2008;36(8):725-730.

13. Patel S, Marshall J, Fitzke FW III. Model for deriving the optical performance of the myopic eye corrected with an intracorneal ring. J Refract Surg 1995;11(4):248-252.

14. Daxer A, Fratzl P. Collagen orientation in the human corneal stroma and its implication in keratoconus. Invest Ophthalmol Vis Sci 1997;38(1):121-129.

15. Alió JL, Piñero DP, Alesón A, et al. Keratoconus-integrated characterization considering anterior corneal aberrations, internal astigmatism, and corneal biomechanics. J Cataract Refract Surg 2011;37(3):552-568.

16. González Pérez J, Cerviño A, Giraldez MJ, et al. Accuracy and precision of EyeSys and Orbscan systems on calibrated spherical test surfaces. Eye Contact Lens 2004;30(2):74-78.

17. Ferrer C, Alió JL, Montañés AU, et al. Causes of intrastromal corneal ring segment explantation: clinicopathologic correlation analysis. J Cataract Refract Surg 2010;36(6):970-977.

18. de Rojas Silva V. Clasificación del queratocono. In: Albertazzi R, ed. Queratocono: pautas para su diagnostico y tratamiento. Buenas Aires, Argentina: Ediciones Científicas Argentinas, 2010:33-97.

19. Tunc Z, Deveci N, Sener B, Bahcecioglu H. Anneaux intracorneéns (Intacs) pour le traitement de l'astigmatisme asymétrique du kératocone: recul de plus de deux ans. J Fr Ophtalmol 2003;26(8):824-830.

20. Colin J, Cochener B, Savary G, Malet F. Correcting keratoconus with intracorneal rings. J Cataract Refract Surg 2000; 26(8):1117-1122.
21. Boxer Wachler BS, Christie JP, Chandra NS, et al. Intacs for keratoconus. Ophthalmology 2003;110(5):1031-1040.

22. Siganos D, Ferrara P, Chatzinikolas K, Bessis N, Bessis N, Papastergiou G. Ferrara intrastromal corneal rings for the correction of keratoconus. J Cataract Refract Surg 2002; 28(11):1947-1951.

23. Zare MA, Hashemi H, Salari MR. Intracorneal ring segment implantation for the management of keratoconus: safety and efficacy. J Cataract Refract Surg 2007;33(11):1886-1891.

24. Kwitko S, Severo NS. Ferrara intracorneal ring segments for keratoconus. J Cataract Refract Surg 2004;30(4):812-820.

25. Piñero DP, Alio JL, El Kady B, et al. Refractive and aberrometric outcomes of intracorneal ring segments for keratoconus: mechanical versus femtosecond-assisted procedures. Ophthalmology 2009;116(9):1675-1687.

26. Kubaloglu A, Sari ES, Cinar Y, Koytak A, Kurnaz E, Ozertürk Y. Intrastromal corneal ring segment implantation for the treatment of keratoconus. Cornea 2011;30(1):11-17.

27. Sansanayudh W, Bahar I, Kumar NL, et al. Intrastromal corneal ring segment SK implantation for moderate to severe keratoconus. J Cataract Refract Surg 2010;36(1):110-113.

28. Torquetti L, Berbel RF, Ferrara P. Long-term follow-up of intrastromal corneal ring segments in keratoconus. J Cataract Refract Surg 2009;35(10):1768-1773.

29. Alió JL, Artola A, Hassanein A, et al. One or 2 Intacs segments for the correction of keratoconus. J Cataract Refract Surg 2005;31(5):943-953.

30. Carrasquillo KG, Rand J, Talamo JH. Intacs for keratoconus and post-LASIK ectasia: mechanical versus femtosecond laser-assisted channel creation. Cornea 2007;26(8):956-962.

31. Piñero DP, Alió JL, El Kady B, Pascual I. Corneal aberrometric and refractive performance of 2 intrastromal corneal ring segment models in early and moderate ectatic disease. J Cataract Refract Surg 2010;36(1):102-109.

32. Piñero DP, Alio JL, Teus MA, Barraquer RI, UcedaMontañés A. Modeling the intracorneal ring segment effect in keratoconus using refractive, keratometric, and corneal aberrometric data. Invest Ophthalmol Vis Sci 2010;51(11): 5583-5591.

33. Kaya V, Utine CA, Karakus SH, Kavadarli I, Yilmaz OF. Refractive and visual outcomes after Intacs vs Ferrara intrastromal corneal ring segment implantation for keratoconus: a comparative study. J Refract Surg 2011;27(12):907-912. 


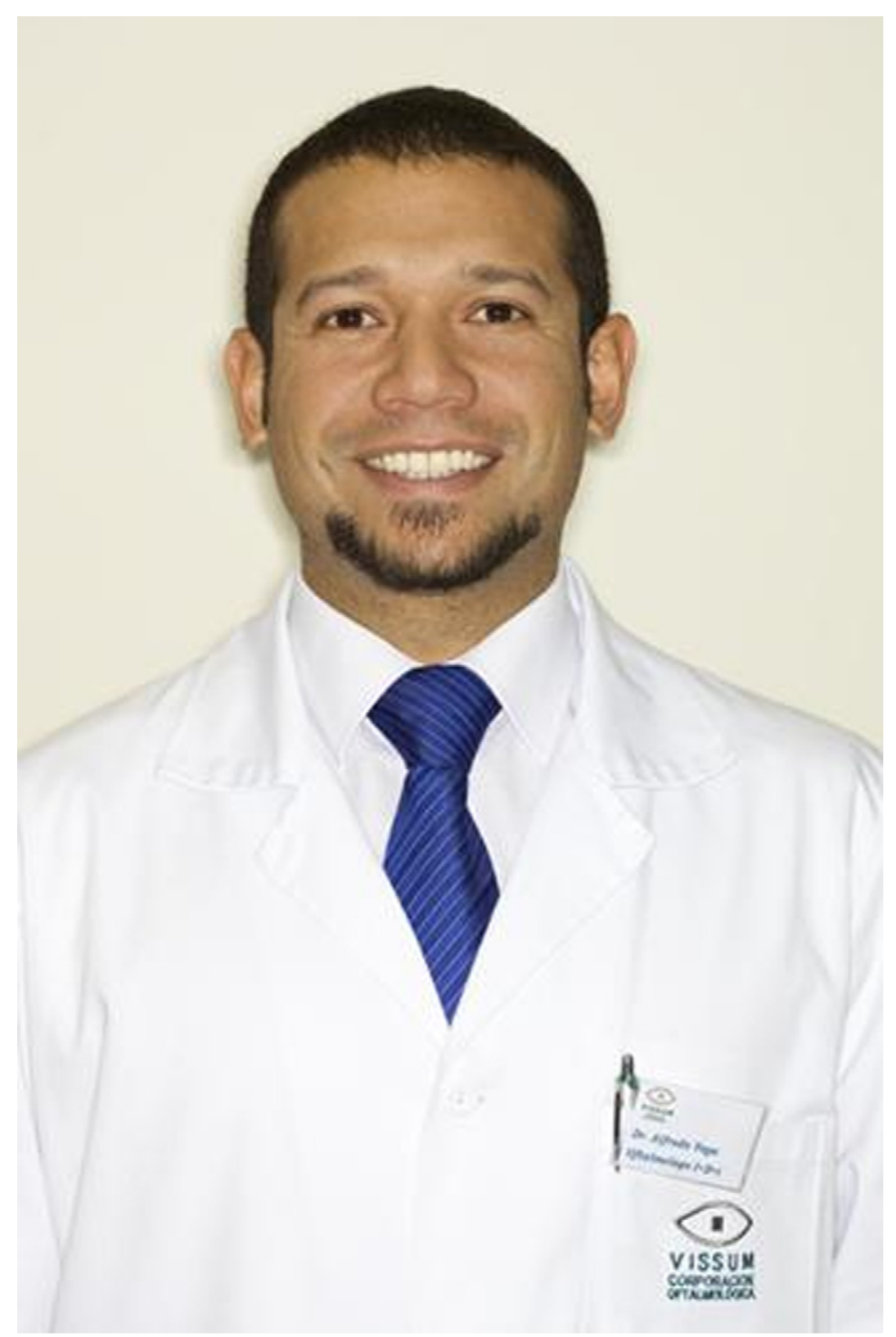

\section{Biosketch}

Alfredo Vega Estrada, MD, MSC, graduated as an ophthalmologist from the Venezuelan Central University and completed his Master's in Clinical and Surgical Research at the Miguel Hernandez University in Spain. Currently, he works in the Cornea and Keratoconus unit at Vissum Corporacion Oftalmologica, Alicante, Spain. Dr Vega Estrada has participated in more than 15 clinical trials and is author of several scientific publications, book chapters and national and international scientific presentations. 